\title{
Relationship between dietary intake and prevalence of obesity in children with down's syndrome
}

\begin{abstract}
This research focuses on the children with Down's syndrome (DS) and how their dietary habits impact their health. The most important aspect of health of these children is the ever-growing rate of obesity prevalent in them and this study seek answers to the question: Are dietary choices of children with Down's syndrome the main perpetrator for overweight and obesity in them? The research took into account 46 students of The Rising Institute and Welfare Society who are suffering from DS between the ages of 6 and 15 years by using non-probability purposive and convenient sampling techniques. The subjects were assessed on their anthropometric measurements like height, weight, Body Mass Index (BMI) and Basal Energy Expenditure (BEE) which was all collected using appropriate tools and formulas. The main task of dietary assessment was done using a 24 Hour Recall and a Food Frequency Questionnaire (FFQ) from the parents/ guardians of the subjects. The results obtained were interpreted and analyzed using SPSS software by applying a Pearson correlation test between the BMI and total energy intake of the subjects. The results obtained were according to the proposed hypothesis and depicted that food choices and energy intake are one of the main reason for prevalence of obesity in children with DS.
\end{abstract}

Volume 9 Issue 2 - 2019

\author{
Angbeen Chaudhary \\ Kinnaird College for Women, Pakistan \\ Correspondence: Angbeen Chaudhary, Kinnaird College \\ for Women, FI 3BSFN027, Pakistan, Tel +923338009800, \\ +965990218I, Email angbeen28637@hotmail.com
}

Received: July 18, 2018 | Published: April 01, 2019

\section{Introduction}

Down's syndrome is a condition that is characterized by mental and growth retardation associated with genetic anomalies, it affects approximately one in 800 live births globally by Aitken \& Spencer, whereas the incidence of Down's syndrome in Pakistan is one in 700 live births by Jamil. There are three types of Down syndrome called Trisomy 21, Translocation and Mosaicism all of which differ in the person's mental ability but have the same characteristics i.e. short stature, broad facial profile, hyper-flexibility, enlarged tongue and obesity. Children with Down's syndrome are more likely to suffer from obesity and overweight. According to a study, 50\% children suffering from Down's syndrome are obese or at a risk of developing obesity in the later years of their life by O'Brien. This alarming rate of obesity development in these children is due to a number of causes like genetics, environmental, social and most importantly dietary. ${ }^{1-6}$

The diet is the main determinant of the weight and health of a child, be it a normal one or a special one. The dietary practices exhibited by children with Down's syndrome are the key to finding out the pattern of obesity development in them. This study will throw light on the dietary practices observed and the development of obesity in the children with Down's syndrome so a relationship can be established between the two variables. Nutritional assessment of the children will be done taking into account the anthropometrics and energy intake in order to understand the connection between the food intake and obesity development in these children. The pattern of obesity is in turn important, so that weight management therapies can be conducted earlier in life to prevent obesity. This research will help to adjust the dietary requirements of children with Down's syndrome, keeping their nutritional and physiological needs in mind, and will also open doors to other studies about special children which will aid parents understand and fulfill the needs of their children in a better way.

\section{Dietary patterns of children with down's syndrome}

Down's syndrome stricken children need a specific diet and nutrient composition to meet their dietary requirements just like ordinary children by Thompson. Their needs are met and addressed by the kind of food they consume, its nutrient composition, frequency of consumption and amount in which it is consumed. The children with Down's syndrome most often have some problems related to the intake, digestion or absorption of food, making their dietary habits even more crucial than normal children by Franco. Diabetes is a usual disease that almost all children with Down's syndrome suffer from after a certain age. Infants with Down syndrome suffer from constipation which is the result of irregular muscle tone and they also have structural abnormalities of the gut which again need surgical interventions. Conditions like these require supervised diet restrictions and recommendations. According to Irish Nutrition, Dietetics Institution, these children must be given additional care by a dietitian to address all the problems and prompt regular growth and development by Calder.

\section{Feeding patterns in children with down's syndrome}

Children especially infants and toddlers with Down's syndrome suffer from a number of drinking and feeding difficulties. They usually arise due to certain characteristic features common to Down's syndrome patients like an abnormally tiny oral cavity, the presence of decreased muscle tone around the muscles of face and a large tongue as a result of high curved palate by Silva. The delay in the tooth development and mouth breathing due to narrow nasal passages result in uncoordinated sucking abilities, irregular swallowing capabilities and difficulty in breathing during feeding. All of these factors can impact on how a child develops efficient oral and feeding skills by Hurson. These disabilities again vary with each individual depending upon the severity of disease as some mothers find it impossible to breastfeed their children or introduce weaning while others have no issues at all. Habitually, weaning or solid food introduction is often delayed in these children when compared to healthy children by Hart. Another feeding problem experienced by Down's syndrome patients is that they suffer from neo-phobia regarding foods that is, inability or fear of trying out new foods due to enhanced oral sensitivities by Dolan. ${ }^{7-12}$ 


\section{Obesity in children with down's syndrome}

A more current apprehension for children suffering from Down syndrome is their propensity to develop obesity in later life. Approximately $17.1 \%$ of children in the U.S. are obese by Ogden. Some research suggests the number of children with Down syndrome who are obese approximates national trends by Cohen. However, the rate of obesity may be much higher than the general population; another study stated that up to $30 \%$ to $50 \%$ of children with Down syndrome are obese by Harris. Fonesca, found children with Down syndrome had an increased risk for developing Type 2 diabetes mellitus due to their propensity for obesity and large amounts of abdominal fat stores by Fonesca. One of the important factor that contributes in the development of obesity in these children is their lifestyle choices and dietary habits. The foods they choose to eat every day play a pivotal role in the growth as well as the trending obesity among them. The growth spurt of adolescents and children born with Down's syndrome is different, rather slow than the other people. Adolescents and adults with Down syndrome tend to be shorter than their peers, and have a lower resting metabolic rate of $10-15 \%$ than the general population which further predisposes to weight gain by Kuperminc. Irish Nutrition and Dietetics Institution recommend that a sensible approach to eating and regular exercise will help to encourage a healthy lifestyle and prevent weight gain in childhood and later life by Wheeler. Using the Food Pyramid as a guide to healthy food choices, choosing appropriate food portions sizes, avoiding excessive amounts of high fat and high sugar foods will help maintain a healthy weight Referral for dietetic intervention may be necessary for guidance with weight loss by Wills.

\section{Methodology}

The study design for this research is correlative as the research takes into account BMI of the subjects and correlates it to their total energy intake. The universe of the study is children with DS and sample included 46 children between the ages of 6 to 15 years enrolled in Rising Sun Institution and Welfare Society for Special Children, Pakistan at the time of research. Convenient and purposive sampling techniques were used, as a school for special children was chosen and all the enrolled students with DS were taken as subjects.

\section{Data collection}

Data collection was a two-step process; the first step involved the collection of anthropometric data like weight, height and age which are important for the proper analysis of Basal Energy Estimate (BEE) of each subject in order to estimate their daily caloric requirement. The BEE was estimated by using Harris-Benedict Equation for males and females. Weight and height are also necessary for determining the Body Mass Index (BMI) of the subject which further helps in determining their nutritional status and obesity. The BMI was calculated by using Quetelet's formula for Body Mass Index i.e. weight in kilograms divided by height in meters squared. The second step of data collection revolved around recording the dietary habits and food choices of the subjects. The dietary intake of the subjects is essential for the evaluation of food consumption of the subjects. This research used a validated Food Frequency Questionnaire (FFQ) provided by Fred Hutchison Research Center, modified on the basis of serving size and food groups to serve the purpose of this research. The dietary intake of subjects was collected by using 24 hour recall, which calculated total daily energy intake made by the subjects. The food intake was reported by the parents/guardians, as the subjects were either non-verbal or too naïve to answer correctly. The 24 hour recall was interpreted by using serving sizes, food groups and total energy intake (BEE) of the subjects for the interpretation of data of this research

\section{Data analysis}

IBM SPSS Statistics 21 was used for the analysis of data for the research. The relationship between BMI and total energy intake was found using Pearson Correlation test on SPSS. ${ }^{12-18}$

\section{Results}

Percentage BMI of subjects depicted that more than $50 \%$ of the subjects were suffering from obesity.

The statistical data of the intake of different food groups like cereals, fruits, vegetables, meat, dairy and fat calculated from the 24 Hour Recall of the subjects is presented below which portrays that among 46 subjects $10.8 \%$ consume fat below recommended intake $23.9 \%$ consume fat according to recommended intake and $65.2 \%$ consume fat above recommended.

The below Figure 1 shows the mean BMI and BEE which is $30.26 \pm 1.99$ and $1090 \pm 177.93$ respectively. The Table 1 also depicts the correlation between BMI and total energy intake of the subjects. The $\mathrm{p}$ value of Pearson Correlation test came out to be 0.006 which is smaller than 0.05 and signifies that energy intake and obesity are dependent on each other.

Table I Correlation between BMI and total energy intake

\begin{tabular}{lllllc}
\hline Variable & $\mathbf{n}$ & Mean & IS.D. & Pearson r value & P value \\
\hline BMI & 46 & 30.26 & 1.99 & - & - \\
Total & & & & & - \\
Energy & 46 & $1090.6 \mathrm{I}$ & 177.93 & - & \\
Intake & & & & & \\
(BEE) & & & & & 0.006 \\
BMI and & & & & $0.40 \mathrm{I}$ & \\
Total & 46 & - & - & & \\
Energy & & & & & \\
Intake & & & & &
\end{tabular}

\section{Body Mass Index}

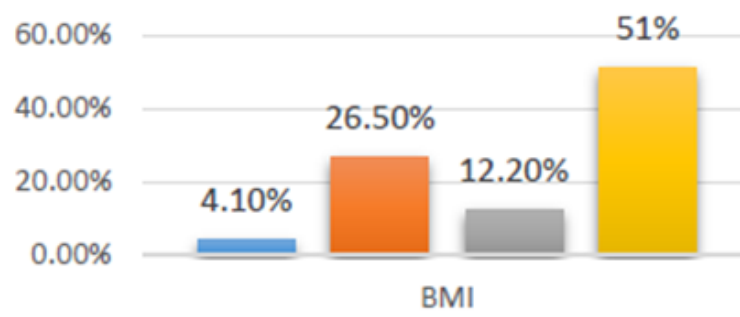

Underweight Normal $\square$ Overweight obese

Figure I Percentage BMI. 


\section{Conclusion}

The BMI of the subjects correlated with the overall consumption of energy. The p-value of the test came out to be less than 0.05 (level of significance) and hypothesis was accepted. It was proved that prevalent obesity in children with Down's syndrome is related to and dependent upon the food choices and total energy intakes of these children. The diets of the subjects were observed to be high in fats and low in fruits, vegetables and fiber. Along with that, a high consumption of fast foods, junk foods and empty calories in the form of sweets and sodas was observed. The total kcal consumption of the children was also greater than their specific recommended intake and thus they were suffering from chronic obesity.

\section{Acknowledgments}

None.

\section{Conflicts of interest}

The authors declare that there is no conflict of interest.

\section{References}

1. Al-Jarallah AS. Down's syndrome and the pattern of congenital heart disease in a community with high parental consanguinity. Med Sci Monit. 2009;15(8):CR409-CR412.

2. Al-Hazzaa HM. Physical activity, fitness and fatness among Saud children and adolescents: implications for cardiovascular health. Saudi Med J. 2002;23(2):144-150.

3. Basil JS, Santoro SL, Martin LJ, et al. Retrospective study of obesity in children with down syndrome. J Pediatr. 2016;173:143-148.

4. Chad K, Jobling A, Frail H. Metabolic rate: a factor in developing obesity in children with Down syndrome? Am J Ment Retard. 1990;95(2):228-235.

5. Collins C, Anne Laverty, Sylvia Roberts, et al. Eating behaviour and food choices in children with Down's syndrome, autistic spectrum disorder or cri du chat syndrome and comparison groups of siblings. Journal of Learning Disabilities. 2003;7(2):137-155.

6. Dietz WH, Robinson TN. Use of the body mass index (BMI) as a measure of overweight in children and adolescents. $J$ Pediatr. 1998;132(2):191-193.
7. Ellis GH. Body Mass Index, physical activity, and working memory in a sample of children with Down syndrome. Regent University Newsletter. 2013;155:3575141.

8. Grammatikopoulou MG, Manai A, Tsigga M, et al. Nutrient intake and anthropometry in children and adolescents with Down syndrome-a preliminary study. Dev Neurorehabil. 2008;11(4):260-267.

9. Hancox RJ, Poulton R. Watching television is associated with childhood obesity: but is it clinically important? Int J Obes (Lond). 2006;30(1):171-175.

10. Hopman E, Csizmadia CG, Bastiani WF, et al. Eating habits of young children with Down syndrome in The Netherlands: adequate nutrient intakes but delayed introduction of solid food. $\mathrm{J} \mathrm{Am} \mathrm{Diet} \mathrm{Assoc.}$ 2008;98(7):790-794.

11. Kawana H, Nonaka K, Takaki H, et al. Obesity and life style of Japanese school children with Down syndrome. Nippon Koshu Eisei Zasshi. 2000;47(1):87-94.

12. Kuczmarski RJ, Ogden CL, Grummer-Strawn LM, et al. CDC growth charts: United States. Adv Data. 2000;314:1-27.

13. Lopes Tde S, Ferreira DM, Pereira RA, et al. Assessment of anthropometric indexes of children and adolescents with Down syndrome. J Pediatr (Rio J). 2008;84(4):350-356.

14. Luke A, Sutton M, Schoeller DA, et al. Nutrient intake and obesity in prepubescent children with Down syndrome. $J$ Am Diet Assoc.1996;96(12):1262-1267.

15. Melville CA, Cooper SA, McGrother CW, et al. Obesity in adults with Down syndrome: a case-control study. J Intellect Disabil Res. 2005;49(Pt 2):125-133.

16. O’Neill KL, Shults J, Stallings VA, et al. Child-feeding practices in children with down syndrome and their siblings. J Pediatr. 2005;146(2):234-238.

17. Samarkandy MM, Mohamed BA, Al-Hamdan AA. Nutritional assessment and obesity in Down syndrome children and their siblings in Saudi Arabia. Saudi Med J. 2012; 33(11):1216-1221.

18. Yang Q, Rasmussen SA, Friedman JM. Mortality associated with Down's syndrome in the USA from 1983 to 1997: a population-based study. Lancet. 2002;359(9311):1019-1025. 